\title{
NDD-01. Animal models to Parkinson's disease study and neuroprotective strategies
}

\author{
ANDRADE, G.M. ${ }^{1}$ \\ ${ }^{1}$ Physiology and Pharmacology Department, Federal University of Ceara
}

Introduction: Parkinson's disease (PD) is a progressive neurodegenerative disorder that is primarily characterized by the degeneration of dopaminergic neurons in the nigrostriatal pathway. It has been suggested that oxidative stress plays a role in the etiology and progression of PD. For instance, low levels of endogenous antioxidants, increased reactive species, augmented dopamine oxidation, and high iron levels have been found in brains from PD patients. Methods: Although the available PD models are imperfect, molecular medicine has been advanced using these models. For example, the MPTP, 6-OHDA and rotenone models have been used to show the efficacy of DA-receptor agonists that are used clinically to reduce PD symptomatology. In addition, the acute in vivo PD models (e.g. the MPTP and nigral 6-OHDA models) and in vitro models have been successfully used to study neuroprotective agents, such as nicotine, estrogen, NO synthase inhibitors and antioxidants, such as polyphenols, coenzyme Q10, and vitamins $A, C$, and $E$. Conclusions: In this presentation, we will review the currently animal models to PD study and show the mechanisms by which polyphenols and endogenous antioxidants can produce protection.

ANDRADE, G.M. 2013. Animal models to Parkinson's disease study and neuroprotective strategies, p.9. In: Oriá, Reinaldo Barreto; Andrade, Geanne Matos de; Bruin, Veralice Meireles S. de. I International Symposium in Neuroscience Meeting [Blucher Neuroscience Proceedings n.1 v.1]. São Paulo: Blucher, 2014 http://dx.doi.org/10.5151/isnm-sine5

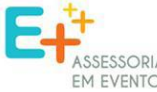

